\title{
Degree Equitable Connected cototal dominating graph
}

\author{
Shigehalli V.S ${ }^{1}$ and Vijayakumar Patil ${ }^{2}$ \\ ${ }^{I}$ (Professor, Department of Mathematics, Rani Channamma University Belagavi, India) \\ ${ }_{2}^{2}$ (Research Scholar, Department of Mathematics, Rani Channamma University Belagavi, India)
}

\begin{abstract}
The degree equitable connected cototal dominating graph $D_{c}^{e}(G)$ of a graph $G=(V, E)$ is a graph with $V\left(D_{c}^{e}(G)\right)=V(G) \cup F$, where $F$ is the set of all minimal degree equitable connected cototal dominating sets of $G$ and with two vertices $u, v \in V\left(D_{c}^{e}(G)\right)$ are adjacent if $u=v$ and $v=D_{c}^{e}$ is a minimal degree equitable connected dominating set of $G$ containing $u$. In this paper we introduce this new graph valued function and obtained some results.
\end{abstract}

Keywords: Connected dominating graph, Degree equitable connected cototal dominating set, Degree equitable connected cototal dominating graph.

\section{Introduction}

The introduction By a graph, we mean a simple and connected. Any undefined terms in this paper may be found in [1].A set $D$ of vertices in a graph $G$ is a dominating set if every vertex not in $D$ is adjacent to at least one vertex in $D$. The domination number $\gamma(G)$ of $\mathrm{G}$ is the minimum cardinality of minimal dominating set of $G[1]$. A dominating set $D$ is said to be a connected dominating set if $\langle D\rangle$ is connected. The connected domination number $\gamma_{c}$ of $G$ is the minimum cardinality of a minimal connected dominating set of $G[6]$. A dominating set $D$ is said to be a cototal dominating set if the induced subgraph $\langle V-D\rangle$ has no isolated vertices. The cototal domination number $\gamma_{\mathrm{cl}}(\mathrm{G})$ of $G$ is the minimum cardinality of a cototal dominating set of $G$ [5].

A subset $D$ of $V$ is called degree equitable dominating set if for every $v \in V-D$ there exist a vertex $u \in D$ such that $u v \in E(G)$ and $|\operatorname{deg}(u)-\operatorname{deg}(v)| \leq 1$, where $\operatorname{deg}(u)$ and $\operatorname{deg}(i)$ denotes the degree of a vertex $u$ and $v$ respectively. The minimum cardinality of such a dominating set is denoted by $\gamma^{e}$ and is called the degree equitable domination number[7].

A connected dominating set $D$ is said to be degree equitable connected co-total dominating set if for every vertex $u \in V-D$ there exist a vertex $v \in<D>$ such that $u v \in E(G)$ and $|\operatorname{deg}(u)-\operatorname{deg}(v)| \leq 1$ provided $\langle V-D\rangle$ contains no isolated vertex. The minimum cardinality of degree equitable connected cototal dominating set is called degree equitable connected cototal domination number of a graph and it is denoted by $\gamma_{c c}^{e}(G)$.

In this paper we define and introduce this new graph valued function and obtained some interesting results.

\section{Definition 1.}

The degree equitable connected cototal dominating graph $D_{c}^{e}(G)$ of a graph $G=(V, E)$ is a graph with $V\left(D_{c}^{e}(G)\right)=V(G) \cup F$, where $\mathrm{F}$ is the set of all minimal degree equitable connected cototal dominating sets of $G$ and with two vertices $u, v \in V\left(D_{c}^{e}(G)\right)$ are adjacent if $u=v$ and $v=D_{c}^{e}$ is a minimal degree equitable connected dominating set of $G$ containing $u$.

\section{Example 1.}

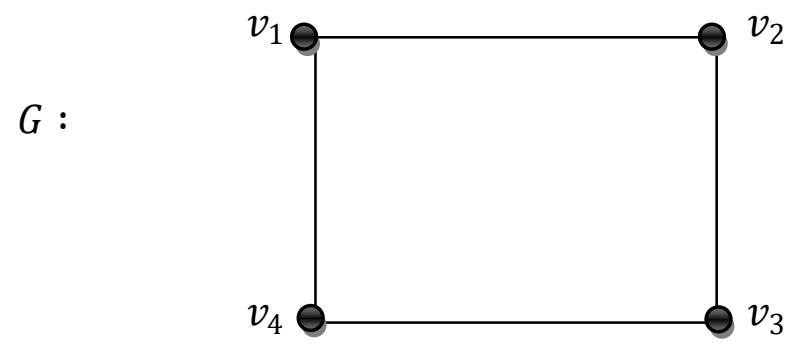

figure. 1 


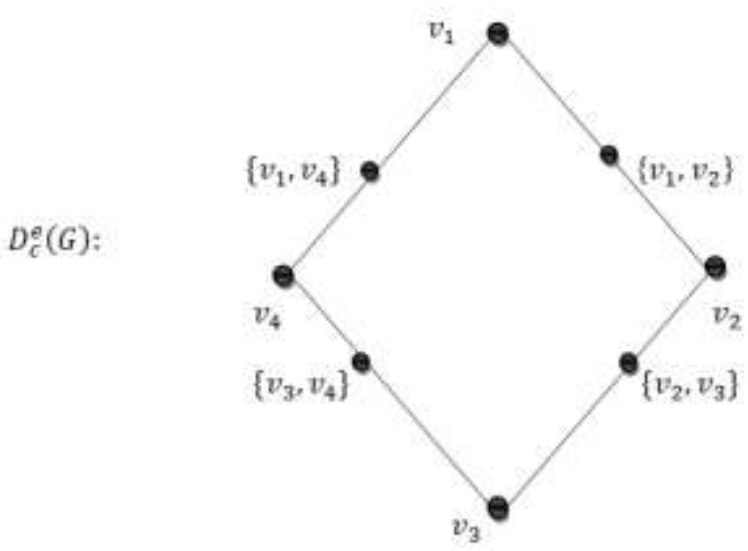

figure. 2

In figure, 1 The dominating set are $D_{1}=\left\{v_{1}, v_{2}\right\}, D_{2}=\left\{v_{1}, v_{4}\right\}, D_{3}=\left\{v_{2}, v_{3}\right\}$ and $D_{4}=\left\{v_{3}, v_{4}\right\}$ and figure. 2 is a degree equitable connected cototal dominating graph.

\section{Observations:}

1) For any graph $G, D_{c}^{e}(G)$ if bipartite.

2) Let $G$ be $(p, q)$ graph, then $\operatorname{deg}_{D_{c}^{e}(G)}\left(v_{i}\right)=$ number of minimal degree equitable dominating set containing $v_{i}$.

\section{Propositions 1:}

For any graph $G, D_{c}^{e}(G) \cong n K_{2}$, if and only if $G=K_{n}, n \geq 3$.

Proof: Let $G=K_{n}, n \geq 3$ be a complete graph of order atleast 3. Since $K_{n}$ is $(n-1)$ - regular graph and each $v_{i} \in V\left(K_{n}\right)$, which act as a the degree equitable connected cototal dominating set. Clearly, $F=\left\{v_{1}, v_{2}, v_{3}, \ldots, v_{n}\right\}$ is a set of all minimal degree equitable connected cototal dominating sets.

Hence, $V\left(D_{c}^{e}(G)\right)=V \cup F$. Further by the construction of degree equitable connected cototal dominating graph. We get the result.

Conversely, Suppose $D_{c}^{e}(G) \cong n K_{2}$ and $G$ is not $K_{n}, n \geq 3$. Then there exist at least one vertex $v \in V(G)$ that appears in atleast two minimal degree equitable connected cototal dominating sets of $\mathrm{G}$.

Hence, in $D_{c}^{e}(G), \operatorname{deg}_{D_{c}^{e}(G)}(v) \geq 2$, a contradiction to our assumption that $G \cong n K_{2}$.

\section{Propositions 2:}

For any graph $G, D_{c}^{e}(G) \cong K_{1, n-1}$ if and only if $G=P_{n}$ or $K_{1, n-1}$ or $K_{m, n}$ if $|m-n| \geq 2$.

Proof: By the earlier results, we know that the degree equitable connected cototal domination number of $P_{n}, K_{1, n-1}, K_{m, n}($ if $|m-n| \geq 1)$ is the entire vertex set of corresponding graph of $G$.

Hence by the construction of $D_{c}^{e}(G)$ we get $D_{c}^{e}(G) \cong K_{1, n-1}$.

Converse is obvious.

\section{Results}

Theorem 2.1: For any graph $G$ with $n \geq 2$ the degree equitable connected cototal dominating graph is connected if and only if $G$ satisfies the following conditions.

i) $\gamma_{c}^{e}(G) \geq 2$.

ii) $\bigcup_{i=1}^{k}\left(D_{c}^{e}\right)=V(G), 1 \leq k \leq n$.

Proof: Let $G$ be any connected graph of order at least two. Suppose $G$ satisfies the hypothesis then there exist a path between every pair of vertex in $D_{c}^{e}(G)$. Hence $D_{c}^{e}(G)$ is connected.

Conversely, suppose $D_{c}^{e}(G)$ is connected and $G$ does not satisfies either of the condition mentioned in the hypothesis. Then $\gamma_{c}^{e}(G)=1$, which means $D_{c}^{e}(G)$ contains a component which is isomorphic to $K_{2}$. 
Further if $\bigcup_{i=1}^{k}\left(D_{c}^{e}\right) \neq V(G)$, then by the definition $D_{c}^{e}(G)$ will be a disconnected graph with atleast two components. Hence by the above two arguments we claim that $D_{c}^{e}(G)$ is disconnected if $D$ does not satisfy the hypothesis of the theorem. Hence the proof.

Theorem 2.2: For any graph $\left.G, \alpha_{0}\left(D_{c}^{e}(G)\right)=\min |\mathrm{W}| V|| F \mid,\right\}$, where $F$ is the set of all minimal degree equitable connected cototal dominating set of $G$.

Proof: Let $V\left(D_{c}^{e}(G)\right)=V \cup F$, By observation $2, V$ and $F$ are independent sets and every element of $V$ is adjacent with some element of $\mathrm{F}$. Also we observe that $|V|<|F|$, then element of $V$ covers all the edges of

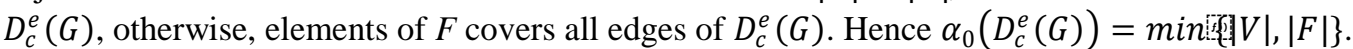

Theorem 2.3: For any graph $\left.G, \beta_{0}\left(D_{c}^{e}(G)\right)=\max |V|,|E|\right\}$, where $F$ is the set of all minimal degree equitable connected cototal dominating set of $G$.

Proof: For any graph $G$, clearly $V$ and $F$ are independent sets of $D_{c}^{e}(G)$. If $|V|>|F|$, then $\beta_{0}\left(D_{c}^{e}(G)\right)=|v|$, otherwise $\beta_{0}\left(D_{c}^{e}(G)\right)=|F|$.

$$
\left.\beta_{0}\left(D_{c}^{e}(G)\right)=\max \text { 需 } V|,| E \mid\right\}
$$

Theorem 2.4: For any graph $G, \chi\left(D_{c}^{e}(G)\right)=2$.

Proof: Let $\mathrm{G}$ be any graph of order at least 2 . By observation 1 . The degree equitable connected cototal dominating graph is bipartite. We use the fact that any bipartite graph is bicolorable.

Hence, $\chi\left(D_{c}^{e}(G)\right)=2$.

Corollary 2.5. For any graph $G, C l\left(D_{c}^{e}(G)\right)=2$, where $C l(G)$ denote the Clique number of $G$.

Proof: Follows from observation 1 and Theorem 2

Theorem 2.6: For any graph G,

$$
\kappa\left(D_{c}^{e}(G)\right)=\min \left[\min _{1 \leq i \leq q} \operatorname{deg}_{D_{c}^{e}(G)}\left|v_{i}\right|, \min _{i \leq j \leq n}\left|F_{j}\right|\right.
$$

Proof: Let $G$ be a $(p, q)$ graph, clearly vertex set and minimal dominating sets of $G$ are independent we consider the following cases,

Case i: Let $x \in v_{i}$ for some $i$, having minimum degree among all $e_{i}$ 's in $D_{c}^{e}(G)$. If the degree of $x$ is less than every other edge in $D_{c}^{e}(G)$, then by deleting those vertices of $D_{c}^{e}(G)$ which are adjacent with $x$, results in a disconnected graph.

Case ii: Let $y \in F_{j}$ for some $j$ having minimum degree among all vertices of $F_{j}$ 's. If degree of $y$ is less than any other vertex in $D_{c}^{e}(G)$. Then by deleting those vertices which are adjacent with $y$, results in a disconnected graph.

$$
\text { Thus, } \kappa\left(D_{c}^{e}(G)\right)=\min ^{\prime} \min _{1 \leq i \leq q} \operatorname{deg}_{D_{c}^{e}(G)}\left|v_{i}\right|, \min _{i \leq j \leq n}\left|F_{j}\right|
$$

Lemma 2.7: For any graph $G$,

$$
\lambda\left(D_{c}^{e}(G)\right)=\min ^{\prime} \min _{1 \leq i \leq q} \operatorname{deg}_{D_{c}^{e}(G)}\left|e_{i}\right|, \min _{i \leq j \leq n}\left|F_{j}\right|
$$

Proof: Let $G$ be a $(p, q)$ graph, clearly vertex set and minimal dominating sets of $G$ are independent, we consider the following cases,

Case i: Let $x \in v_{i}$ for some $i$, having minimum degree among all $e_{i}$ 's in $D_{c}^{e}(G)$. If the degree of $x$ is less than every other edge in $D_{c}^{e}(G)$, then by deleting those vertices of $D_{c}^{e}(G)$ which are adjacent with $x$, results in a disconnected graph.

Case ii: Let $y \in F_{j}$ for some $j$ having minimum degree among all vertices of $F_{j}$ 's. If degree of $y$ is less than any other vertex in $D_{c}^{e}(G)$. Then by deleting those vertices which are adjacent with $y$, results in a disconnected graph.

$$
\text { Thus, } \lambda\left(D_{c}^{e}(G)\right)=\min _{\left(\min _{1 \leq i \leq q}\right.} \operatorname{deg}_{D_{c}^{e}(G)}\left|e_{i}\right|, \min _{i \leq j \leq n}\left|F_{j}\right|
$$

Theorem2.8: For any graph $G, \gamma\left(D_{c}^{e}(G)\right)=1$ if and only if $G$ contains a minimal dominating set of cardinality $n$.

Proof: Suppose $G$ contains a minimal degree equitable connected cototal dominating set of cardinality $n$, then $D_{c}^{e}(G)$ contains a vertex of degree $(n-1)$. Hence $\gamma\left(D_{c}^{e}(G)\right)=1$. 
Conversely, suppose $\gamma\left(D_{c}^{e}(G)\right)=1$ and $\mathrm{G}$ does not contains a minimal degree equitable connected cototal set of cardinality $n$, then by construction of $D_{c}^{e}(G)$ contains vertex of degree less then $n-1$, which implies $\gamma\left(D_{c}^{e}(G)\right)>1$ a contradiction.

Theorem2.9: For any graph $G, \gamma\left(D_{c}^{e}(G)\right)=n$ if and only if $G=K_{n}, n \geq 3$.

Proof: Let $G=K_{n}, n \geq 3$, then by proposition $1, D_{c}^{e}(G) \cong n K_{2}$.

Further, we know that $\gamma\left(n K_{2}\right)=\frac{n}{2}$, Since $\left|V\left(D_{c}^{e}\left(K_{n}\right)\right)\right|=2 n$,

Hence $\gamma\left(D_{c}^{e}\left(K_{n}\right)\right)=n$.

Converse is obvious.

Theorem2.10: For any graph $G, D_{c}^{e}(G)$ is cycle if and only if $G=C_{4}$.

Proof: Let $G=C_{4}$ be a cycle of a length four, then we get exactly four degree equitable connected dominating sets of cardinality two and every vertex of $C_{4}$ is present in exactly two minimal degree equitable connected cototal dominating sets. Hence by the definition $D_{c}^{e}(G)$ every vertex in $D_{c}^{e}\left(C_{4}\right)$ having degree two. That is $D_{c}^{e}\left(C_{4}\right)$ is a connected 2 - regular graph.

Hence, $D_{c}^{e}\left(C_{4}\right) \cong C_{8}$.

Conversely, suppose $D_{c}^{e}\left(C_{4}\right)$ is a cycle and $G$ is not isomorphic to $C_{4}$. Then $G$ contains atleast one vertex which is present in either one minimal degree equitable connected cototal dominating set or presents in atleast three minimal equitable connected cototal dominating sets.

Hence by the definition of $D_{c}^{e}(G)$ the degree of corresponding vertex is one or atleast three, a contradiction.

Theorem A[1]: A graph $G$ is Eulerian if and only if degree of every vertex is even.

Theorem B[1]: Let $G=(V, E)$ be a graph, if $V(G)=V_{1} \cup V_{2}$ with $\left|V_{1}\right|=\left|V_{2}\right|$ then $G$ is Hamiltonian.

Theorem2.11: For any graph $G$ with $\gamma(G) \geq 2$ and $\cup_{i=1}^{k} D_{c}^{e}(G)=V(G)$ then $D_{c}^{e}(G)$ is Eulerian, if $G$ satisfy the following condition.

i) Every vertex $v_{i}=V(G)$ is presenting even number of minimal degree equitable dominating sets of $G$.

ii) The cardinality of every minimal degree equitable connected cototal dominating set must be even.

Proof: Suppose $\gamma(G) \geq 2$ and $\bigcup_{i=1}^{k} D_{c}^{e}(G)=V(G)$ then by Theorem $1, D_{c}^{e}(G)$ is connected. Hence, now we need to show that $D_{c}^{e}(G)$ is Eulerian.

Suppose, $G$ satisfies condition (i) and (ii). Hence, degree of every vertex in $D_{c}^{e}(G)$ is even therefore by Theorem (A), $D_{c}^{e}(G)$ is Eulerian.

Conversely, suppose $D_{c}^{e}(G)$ is Eulerian and $G$ does not satisfies condition (i) and (ii), then $D_{c}^{e}(G)$ contains a vertex of odd degree. Hence by Theorem A, $D_{c}^{e}(G)$ is not Eulerian, which is a contradiction.

Theorem2.12: Let $G=(V, E)$ be a graph if $V\left(D_{c}^{e}(G)\right)=V_{1} \cup V_{2}$ with $\left|V_{1}\right|=\left|V_{2}\right|$ then $D_{c}^{e}(G)$ is Hamiltonian. Proof: Let $G$ be a bipartite graph, by observation (1), we know that $D_{c}^{e}(G)$ is bipartite. If $V\left(D_{c}^{e}(G)\right)=V_{1} \cup V_{2}$ with $\left|V_{1}\right|=\left|V_{2}\right|$ then by Theorem B, $D_{c}^{e}(G)$ is Hamiltonian.

Conversely, Suppose $V\left(D_{c}^{e}(G)\right)=V_{1} \cup V_{2}$ with $\left|V_{1}\right| \neq\left|V_{2}\right|$ then $G$ does not contain a Hamiltonian cycle, a contradiction.

\section{References}

[1] F. Harary, Graph Theory, Addison-Wesley, Reading Mass,(1969).

[2] T. W. Haynes, S.T. Hedetniemi, and P.J.Slater, Fundamentals of domination in graphs, Marcel Dekker, Inc, New York, (1998).

[3] E. J. Cockayne and S. T. Hedetniemi, Towards a theory of domination in Graphs, Networks, (7)(1977),247-26.

[4] B. Basavanagoud and S. M. Hosamani, Degree equitable connected domination in graphs, ADMS, 5(1) (2013), 1-11

[5] V. R. Kulli, B. Janakiram and R. R. Iyer, The cototal domination of graph, Discrete Mathematical Sciences and Cryptography 2 (1999), 179-184. 
[6] E. Sampathkumar and H. walikar, The connected domination number of a graph, Journal of Mathematical and Physical Sciences 13(1979), 607-613.

[7] V. Swaminathan and K. M Dharmalingam, Degree equitable domination on graph, Kragujevac Journal of Mahtematics,1(35) (2011), s191-197.

[8] Shigehalli V.S and Vijayakumar Patil, Degree equitable connected cototal domination number of graph, International Journal of Mathematical Archive 7-11 (2016) 96-100. 